\title{
Sequence Tolerance of a Single-Domain Antibody with High Thermal Stability: Comparison of Computational and Experimental Fitness Profiles
}

Mark A. Olson, ${ }^{* \dagger}$ Patricia M. Legler,${ }^{\ddagger}$ Daniel Zabetakis, ${ }^{\ddagger}$ Kendrick B. Turner, George P. Anderson and Ellen R. Goldman

${ }^{\dagger}$ Systems and Structural Biology Division, USAMRIID, Frederick, Maryland, United States of America

\$Center for Bio/Molecular Science and Engineering, Naval Research Laboratory, 4555 Overlook Ave SW, Washington, D.C., United States of America 
Figure S1. Representative size exclusion chromatography (SEC) data obtained from purification of the sdAbs. The major peak was collected and used in the SPR and CD experiments. The A3 wild-type (WT) and all of the A3 mutants characterized in this manuscript were expressed periplasmically and were purified by immobilized metal affinity chromatography (IMAC) followed by SEC. As seen in the representatives below, all of them were majority monomer following IMAC purification. We purified and studied only the monomeric fraction obtained from the SEC purification. We have seen no evidence under the conditions we maintained the sdAb that the A3 monomer or any of the purified monomer variants forms dimers.

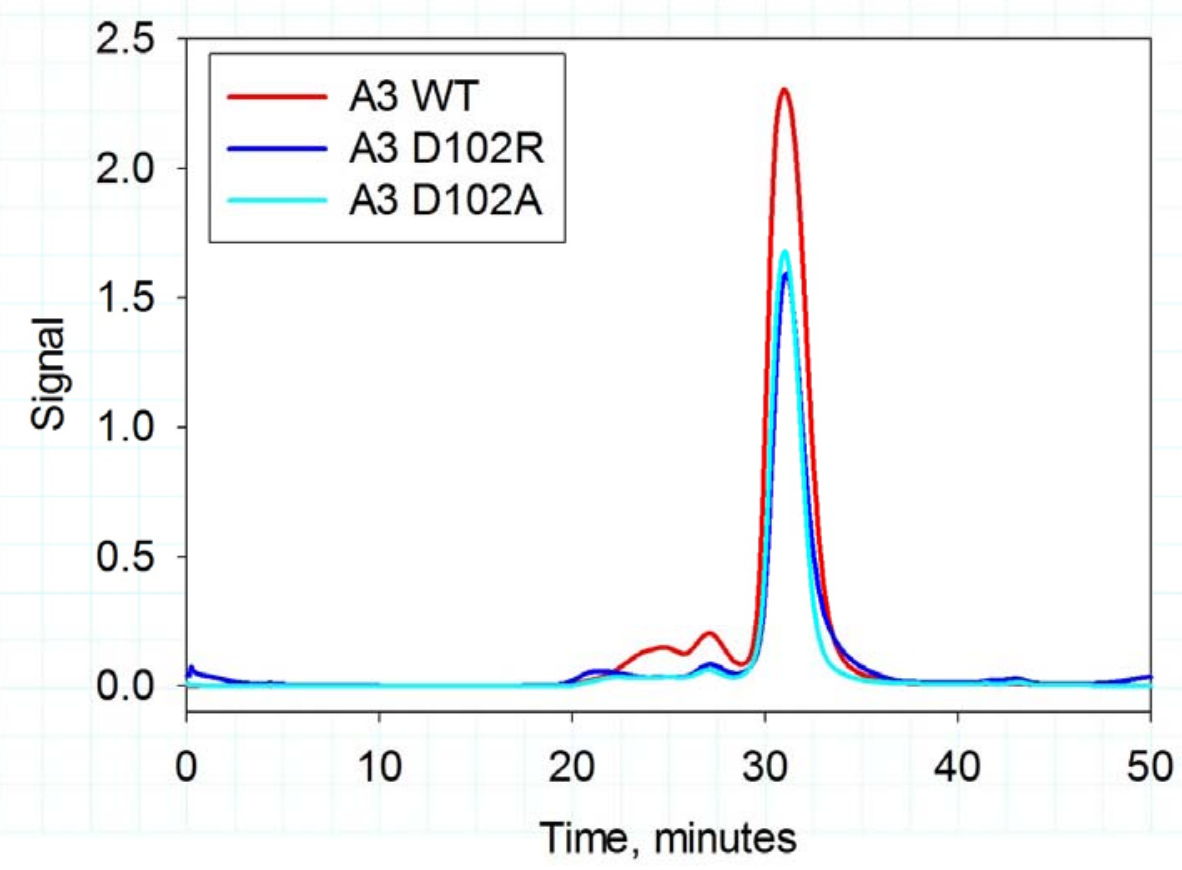


Figure S2. Representative SPR data from A3 wild-type (WT) and mutants. In each case the numbers to the right of the curve represent the concentration of the $s d A b$ in $n M$. Above each set of traces the values for $k_{a}, k_{d}$, and $K_{D}$ are shown. Other than the mutants at P55, the $K_{D}$ values of the mutants are considered essentially equivalent to wild-type

All the SPR data was from samples of A3 WT and A3 variants that were purified as monomer and no dimer or aggregate was present in any sample.

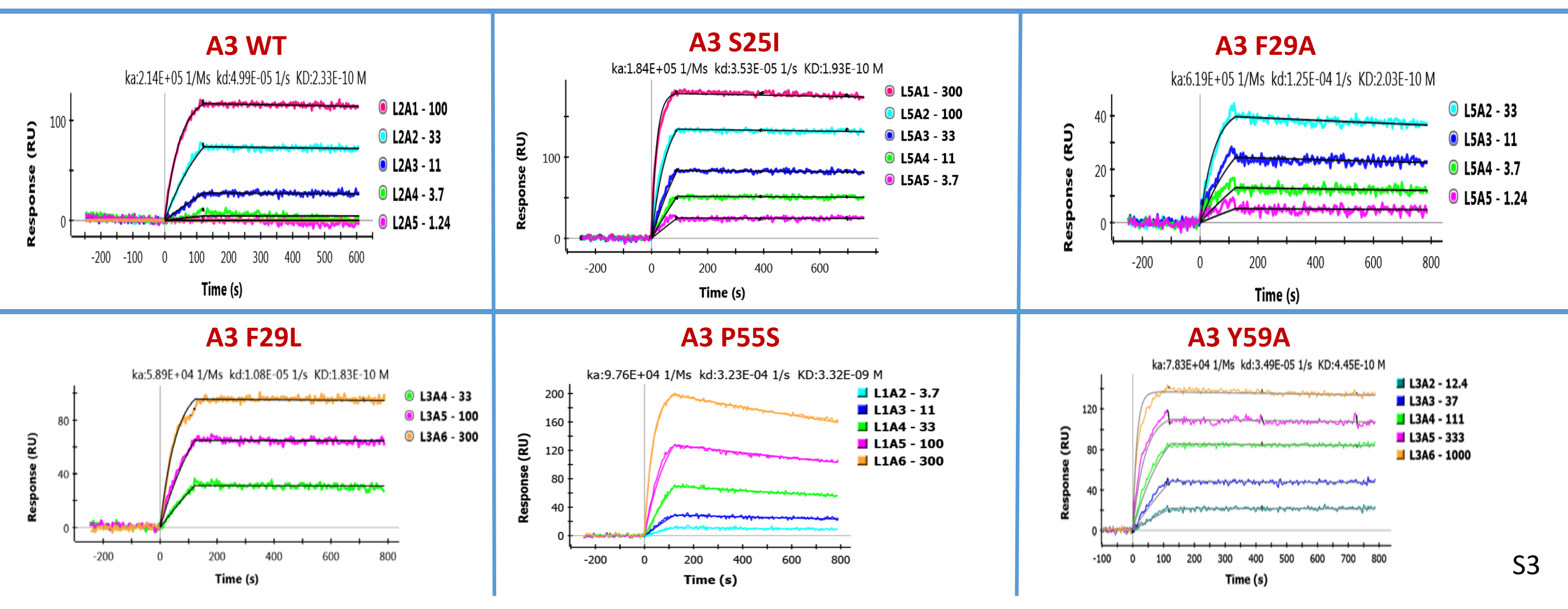




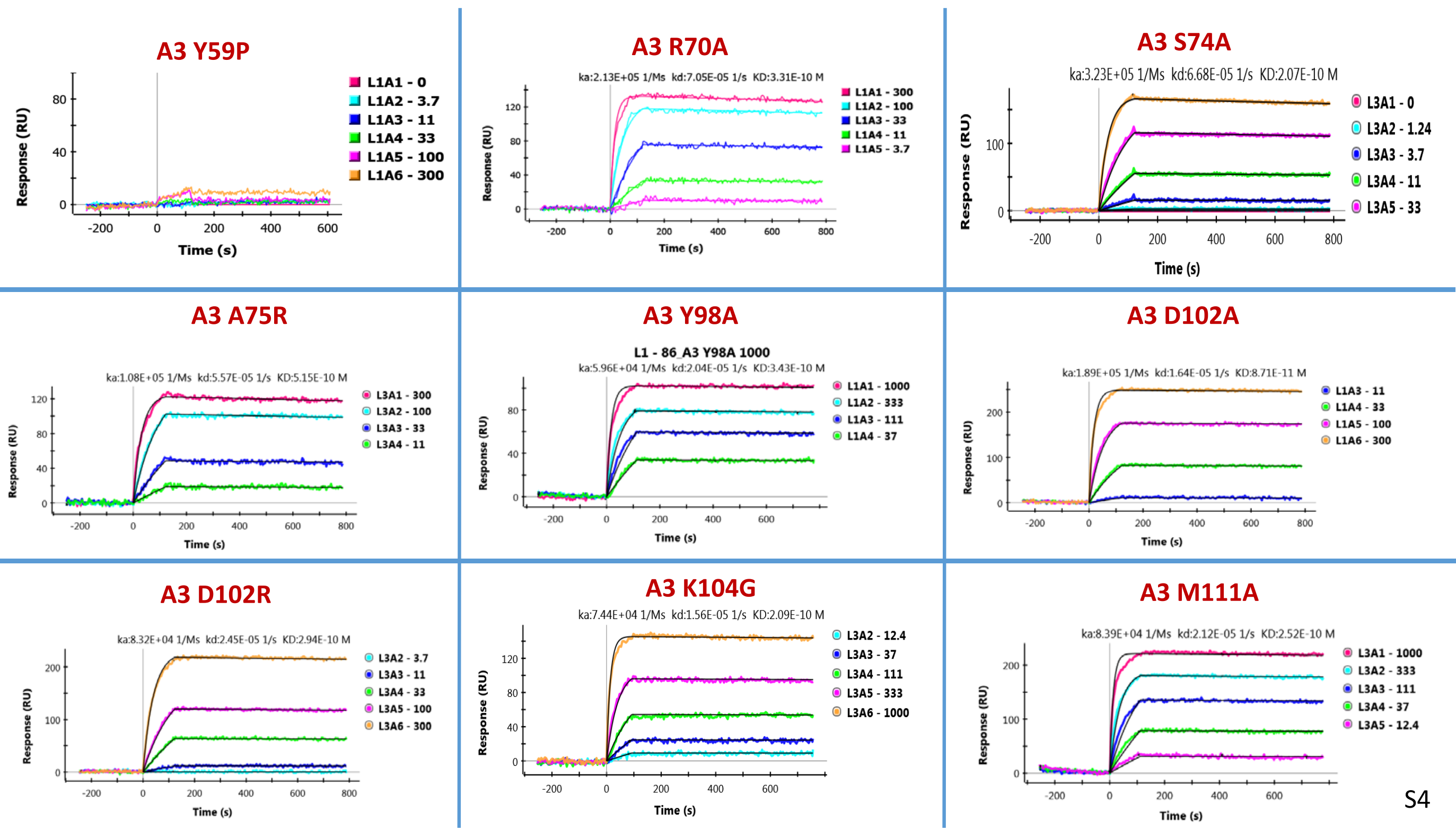



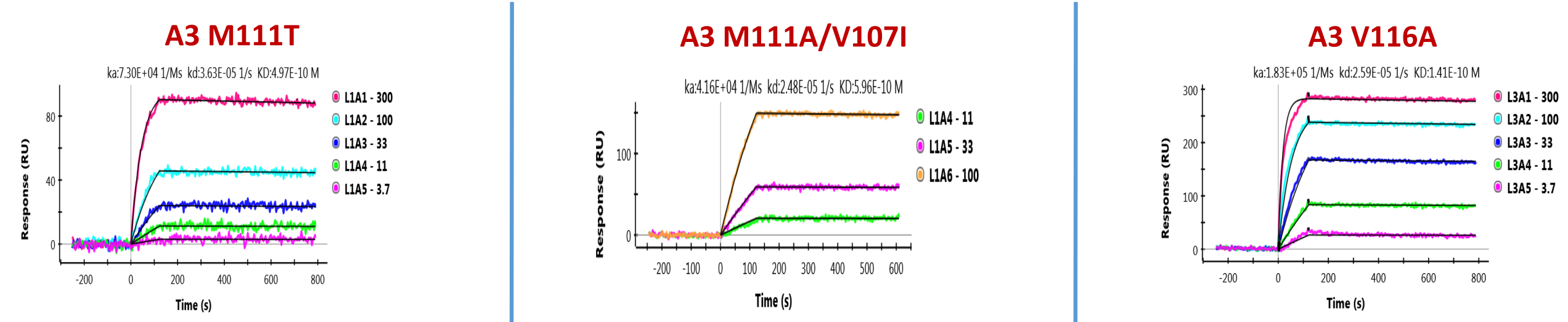

\section{A3 V116Y}

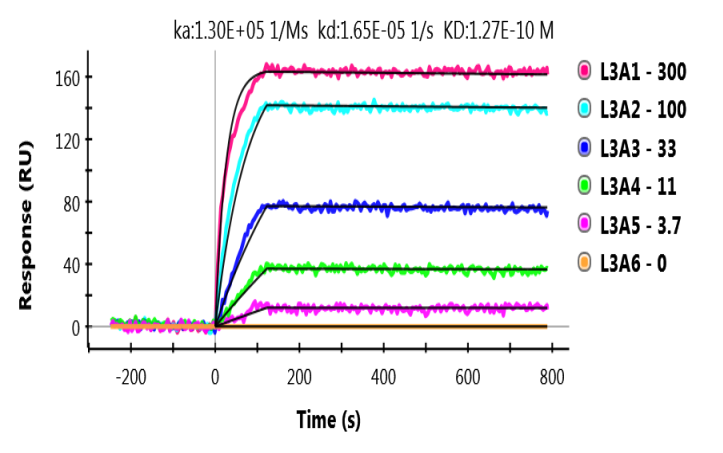


Figure S3. Representative CD data from A3 wild-type (WT) and mutants. Melting temperatures are noted by the blue line in each plot. All the $C D$ data was from samples of $A 3$ and $A 3$ variants that were purified as monomer and no dimer or aggregate was present in any sample.
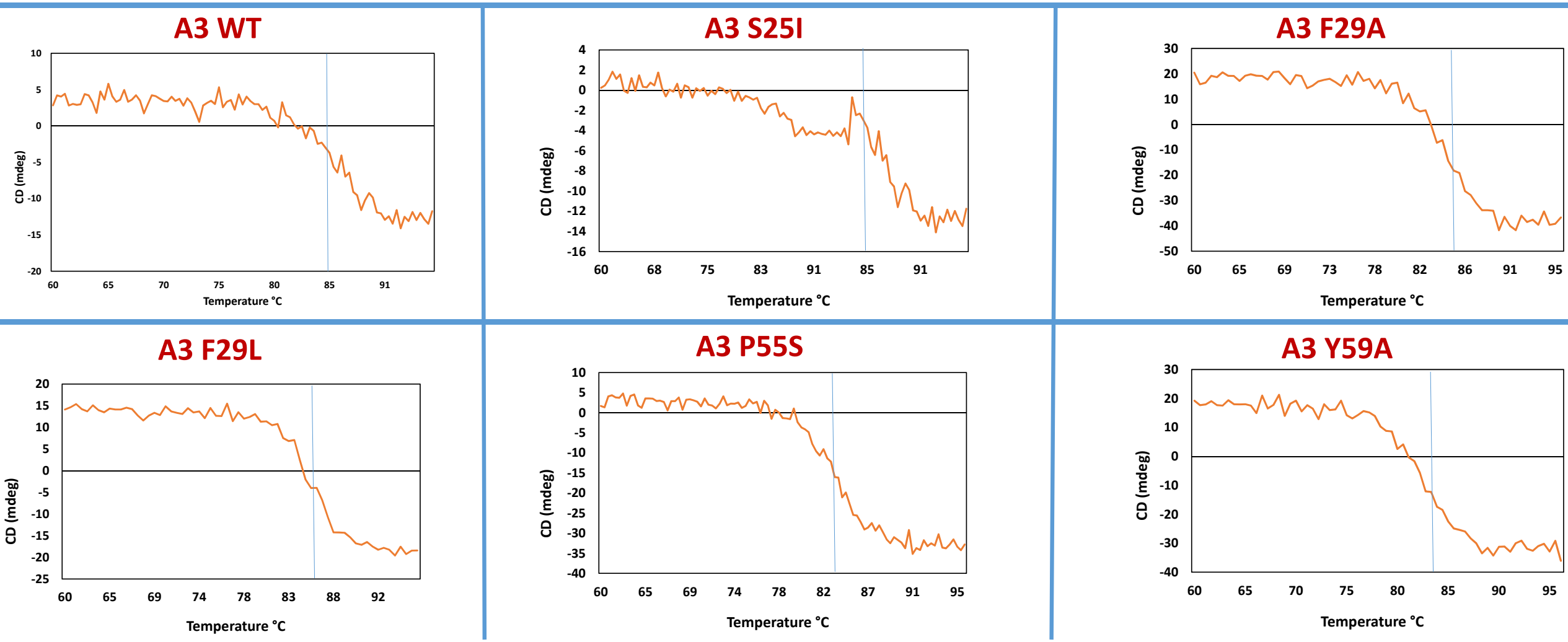

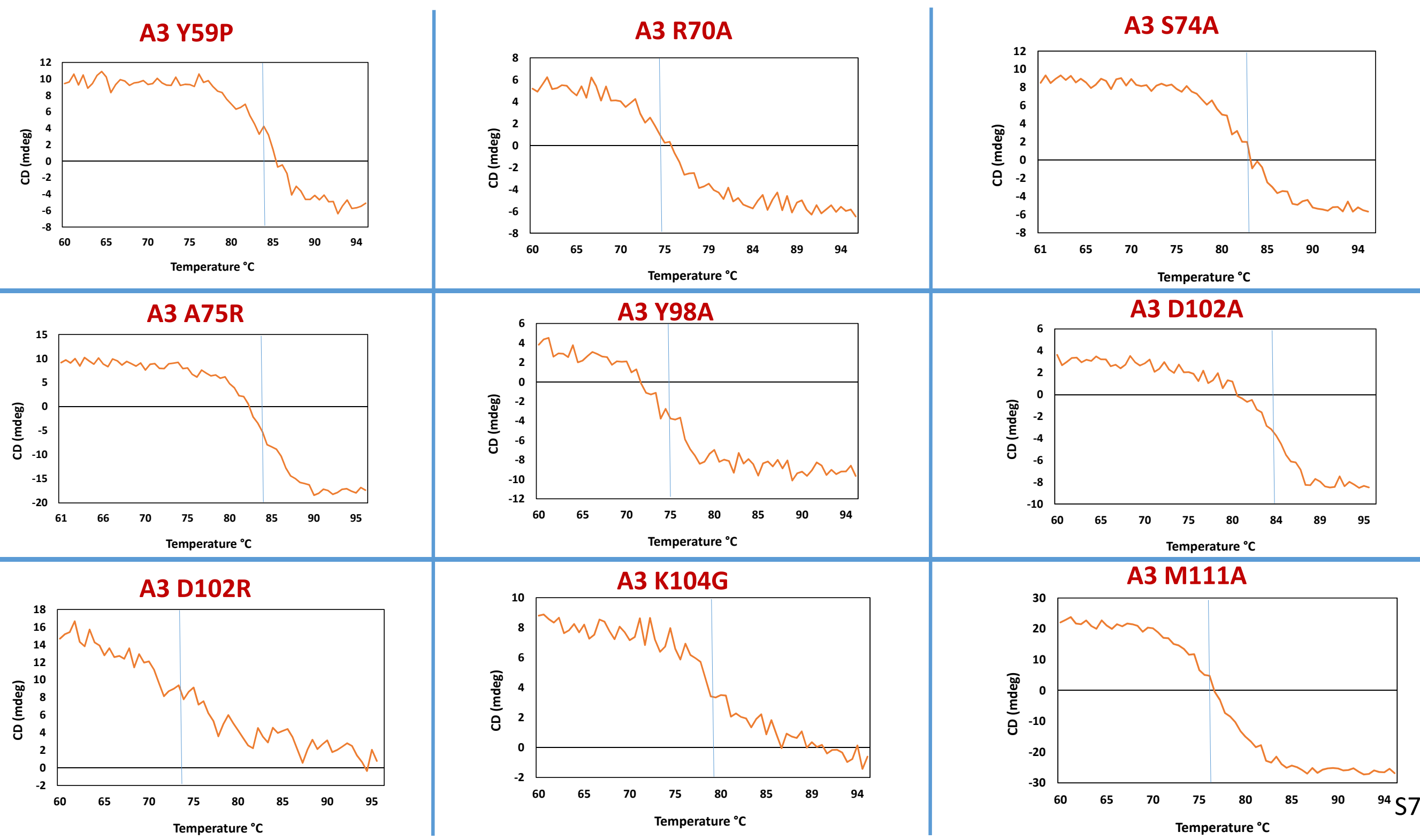

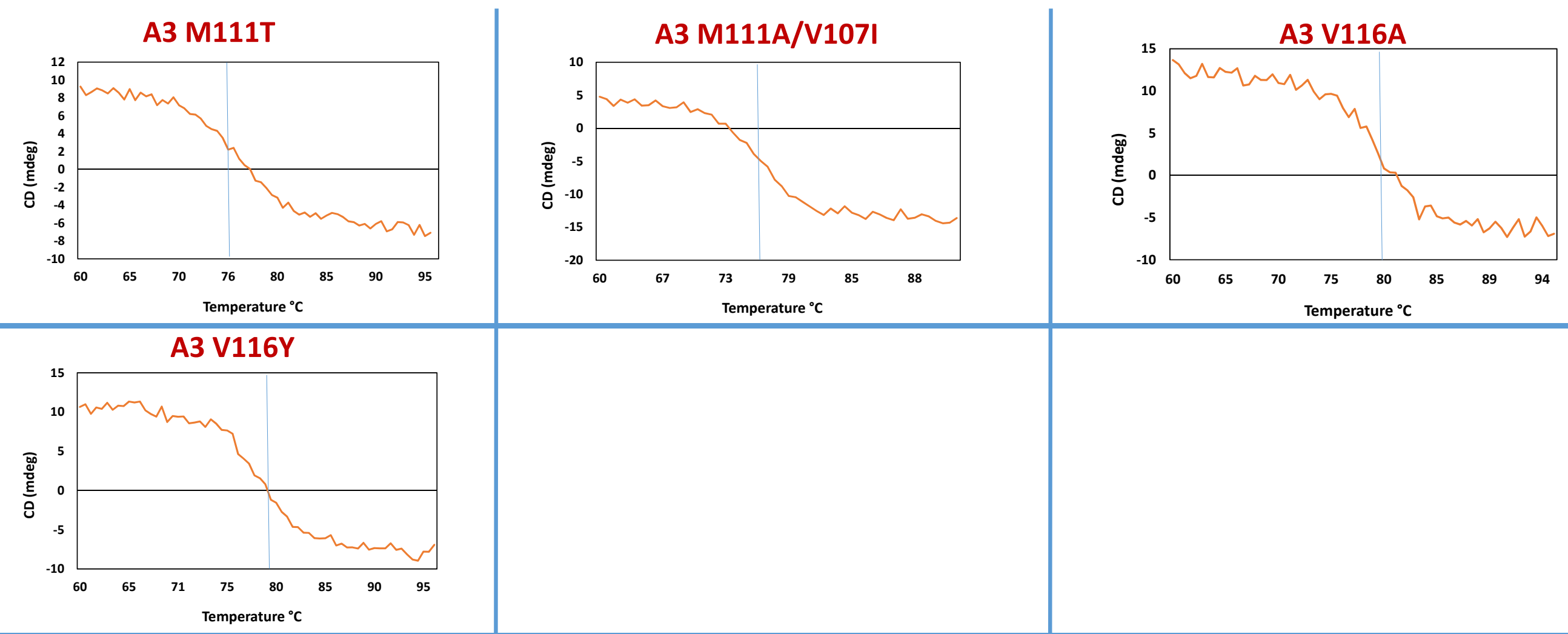